\title{
Appearance of high-temperature phase in zirconia films made by aerosol gas deposition method
}

\author{
Eiji FUCHITA, ${ }^{* \dagger} \dagger$ Eiji TOKIZAKI, ${ }^{*}$ Eiichi OZAWA* and Yoshio SAKKA
}

National Institute for Materials Science, 1-2-1 Sengen, Tsukuba, Ibaraki 305-0047

*Fuchita Nanotechnology, Ltd., 2-25-57 Tamatsukuri, Narita, Chiba 286-0011

The aerosol gas deposition method (AGD) is a low-temperature method. No heating occurs during the AGD process when forming the AGD films. Nevertheless, ceramic films can be synthesized at room temperature directly from the source ceramic powders. In this paper, we report that the high-temperature phase of zirconia was found in zirconia films synthesized by AGD and that a light emission phenomenon was observed at the deposition site. The generation of the high-temperature phase was dependent on the mean diameter of the source ceramic powders. Both a small powder of less than two or three microns in diameter and large powder particles of more than 10 microns in diameter have the low possibility of generating the high-temperature phase when compared to that of 7-micron diameter powder. The appearance of the high-temperature phase and the light emission might be related to the formation mechanism of the AGD film at ambient temperature.

(C)2011 The Ceramic Society of Japan. All rights reserved.

Key-words : High-temperature phase, Light emission, Zirconia powder, Aerosol gas deposition, Film formation, Mean particle size

[Received November 8, 2010; Accepted December 28, 2010]

\section{Introduction}

The aerosol gas deposition method is considered to be a totally low-temperature process. ${ }^{1)-7)}$ Even at room temperature, a ceramic powder can be solidified and form a thin film. From a thermodynamic point of view, these phenomena are difficult to understand. People have tried to clarify the consolidation mechanism of the AGD films. ${ }^{3), 4)}$ However, no clear explanation of the mechanism has been offered until now. What is known follows. The configurations (for instance, size, specific surface, dryness, etc.) of the source ceramic powder significantly determines whether thin ceramic films can be successfully synthesized from the source powder. As a result, some preliminary treatments, such as milling and drying of commercially obtained powders, are suggested before synthesizing the AGD films. From SEM and TEM observations, it is known that the synthesized films are composed of fine particles of less than one-fifth to one-hundredth of the diameter of the source powders. The question is how the crushed powder is related to the sintering of the source powder. Part of the kinetic energy of the ejected powder from a nozzle should be consumed during crushing of the starting powder, and the total energy itself is not sufficient to cause the powder to sinter for ceramic film formation on a substrate. The temperature change of the substrate was only 2 or 3 degrees Centigrade according to the thermocouple measurement. Consequently, it was obvious that we should find another driving force to consolidate the ceramic powders at room temperature.

Under these circumstances, we tried to synthesize AGD films with the powders that were considered to be difficult to form AGD films. We selected zirconia powder because of its high potential for industrial use. If we can determine the reason why zirconia is difficult to use in making films by AGD, we might

\footnotetext{
Corresponding author: E. Fuchita; E-mail: fuchita@nanotechjp. com
}

acquire some understanding about the AGD film formation mechanism. We have published two papers on zirconia film formation. ${ }^{8), 9)}$ In these two papers, we reported that an AGD film using zirconia could be synthesized when we used powders having a mean diameter in the micron range $(0.73$ to $10.2 \mu \mathrm{m})$ and the special specific surface areas were from 1.5 to $6.1 \mathrm{~m}^{2} / \mathrm{g}$. These diameters were one order greater than those shown in the past papers. ${ }^{3)-7)}$ We also observed new phenomena involving the high-temperature phase of zirconia present in the XRD data of the obtained AGD films and stable luminescent characteristics at the impact site of the ejected powder on a substrate. In this paper, we discuss the high-temperature phase of zirconia correlated to the size of the source powder and light emission from the deposition site of the zirconia.

\section{Experimental procedure}

\subsection{Materials used}

Commercially available zirconia powders (Daiichi Kigenso Kagaku Kogyo Co., Ltd.) were used as the starting powders, the same as in our past studies. Table 1 shows the five powders made by a dry method and one powder made by a wet method. The powders labeled TMZ-T to BR-12QZ were made by the dry method and SPZ was from the wet method. Their particle sizes were in the range of 0.73 to $10.2 \mu \mathrm{m}$ and their specific surface areas ranged from 1.5 to $6.1 \mathrm{~m}^{2} / \mathrm{g}$.

\subsection{Experimental procedure}

The AGD setup used in this study was very similar to the old introduced in the previous papers. ${ }^{8), 9)}$ Figure 1 is a schematic diagram of the AGD apparatus, which is composed of an aerosol container, a deposition chamber, and transfer tubing. The chamber is first evacuated, then nitrogen gas is introduced into the chamber. The gas is then pumped to the deposition chamber through the transfer tubing. The zirconia powder encased in the aerosol container is entrained by the supply gas and ejected out of the nozzle and into the deposition chamber. The experimental 


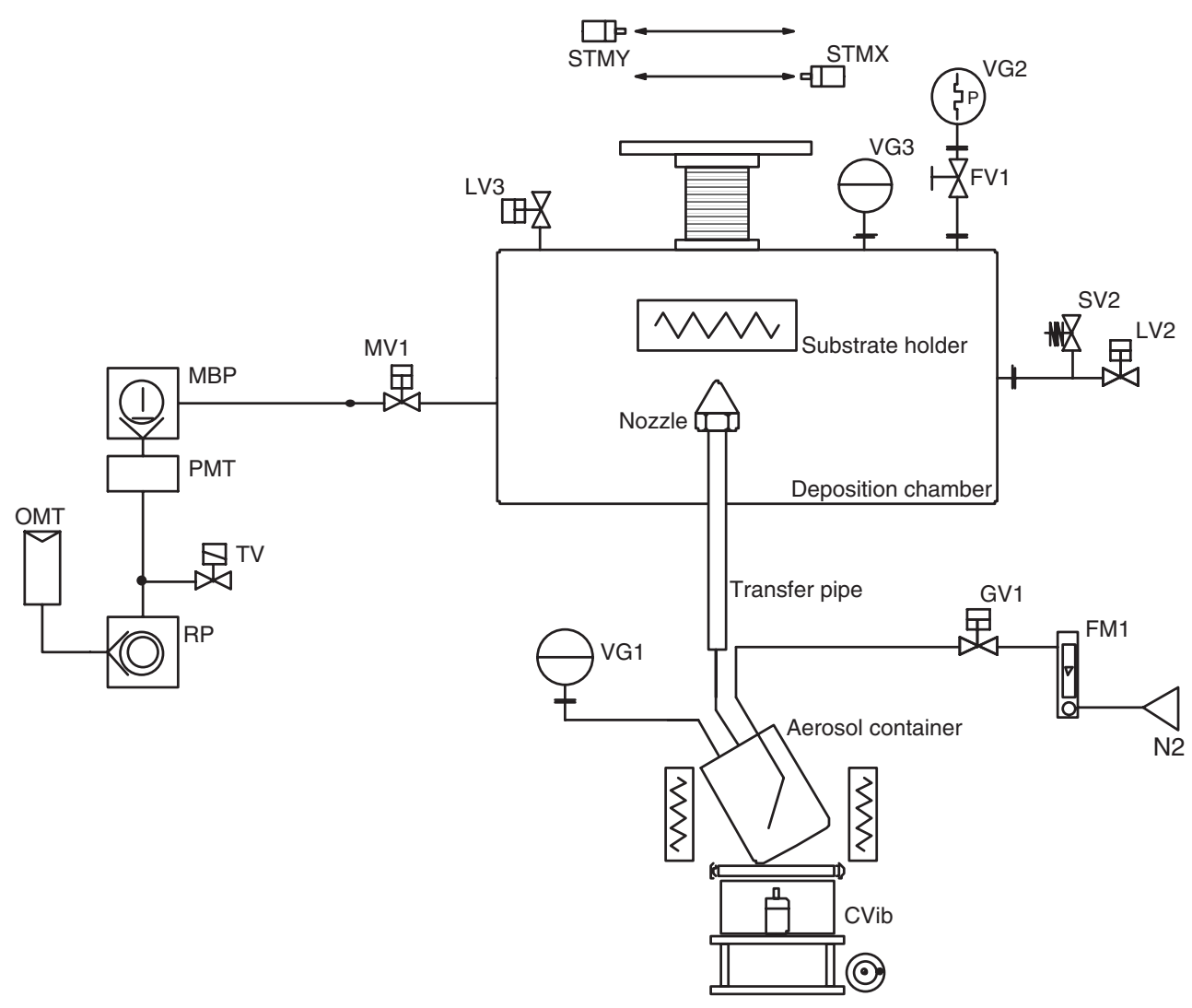

Fig. 1. Schematic diagram of the AGD apparatus.

Table 1. Characteristics of zirconia powders

\begin{tabular}{cccccccc}
\hline & $\begin{array}{c}\text { Mean } \\
\text { particle } \\
\text { size } D_{50} \\
\mu \mathrm{m}\end{array}$ & $\begin{array}{c}\text { Specific } \\
\text { surface } \\
\text { area } \\
\mathrm{m}^{2} / \mathrm{g}\end{array}$ & $\begin{array}{c}\mathrm{ZrO}_{2}+\mathrm{HfO}_{2} \\
\%\end{array}$ & $\begin{array}{c}\mathrm{SiO}_{2} \\
\%\end{array}$ & $\begin{array}{c}\mathrm{Fe}_{2} \mathrm{O}_{3} \\
\%\end{array}$ & $\begin{array}{c}\mathrm{TiO}_{2} \\
\%\end{array}$ & Product \\
& 0.73 & 6.1 & Main elements & 0.14 & 0.12 & 0.17 & TMZ-T \\
1 & 0.12 & 4.7 & Main elements & 0.07 & 0.08 & 0.15 & TMZ \\
2 & 1.12 & 2.7 & Main elements & 0.18 & 0.16 & 0.17 & BR-3QZ \\
3 & 2.9 & 1.6 & Main elements & 0.12 & 0.05 & 0.18 & BR-QZ \\
4 & 7.4 & 1.5 & Main elements & 0.19 & 0.04 & 0.21 & BR-12QZ \\
5 & 10.2 & 1.2 & Main elements & 0.001 & 0.003 & 0.003 & SPZ \\
6 & 3.5 & 6.1 & & & & & \\
\hline
\end{tabular}

conditions were completely the same as in the previous studies. Each powder was heat-treated at $773 \mathrm{~K}$ for $1 \mathrm{~h}$ before its use. The nozzle had a width of $30 \mathrm{~mm}$ and a $0.3-\mathrm{mm}$-wide slit. Glass plates used as the substrates were $70 \mathrm{~mm}$ (length) $\times 50 \mathrm{~mm}$ (width) $\times 1 \mathrm{~mm}$ (thickness). The pressure of the aerosol container was varied from 22 to $42 \mathrm{kPa}$ (differential pressure). The nitrogen gas flow was from 4 to $16 \mathrm{~L} / \mathrm{min}$ during the film formation procedure. Scanning was performed from 50 to 1000 times along the $X$ direction to obtain the final film. The entire deposition process was carried out at room temperature.

XRD and SEM were used for characterization of the AGD films. A compact digital video camera was also used to observe the deposition progress at the deposition site on the glass substrate.

\section{Results and discussion}

\subsection{XRD micrographs}

Figures 2(a)-2(f) show XRD micrographs of the 6 powders. All the deposited films were found to be composed of a mono- clinic structure and a high-temperature phase with a tetragonal structure and a cubic one, although all the starting powders do not contain any high temperature phases. Figure 3 shows their Raman spectra. In the spectra, a monoclinic structure and a tetragonal structure can be identified. However, the cubic structure cannot be identified. Therefore, the diffraction peaks from the high temperature phase will be those of the tetragonal structure. Figure 2(d) shows the XRD data for the starting powder and the AGD film made from the BR-QZ powder. In the XRD micrographs of the BR-QZ AGD films, the (101) peak of the tetragonal structure is clearly observed and both their (002) and (110) peaks seem to overlap the peaks of the monoclinic structure. The AGD films contain the tetragonal phase as if they were heated at more than about one thousand degrees Centigrade, then rapidly quenched.

The amount of the tetragonal structure in the AGD films seems to depend on the starting powders. Figure 4 shows the result of a quantitative analysis of the BR-QZ using the Rigaku's PDXL software. Table 2 shows the ratio of the strongest peak of the tetragonal structure and the monoclinic structure of the AGD films made from the 6 starting powders. The BR-QZ powder contains the highest amount of the tetragonal structure, while the TMZ-T one has the lowest amount of the tetragonal structure. Figure 5 shows the relation between the tetragonal-to-monoclinic structure ratio and the mean diameter of the starting powders. Why can BR-QZ generate the highest amount of the tetragonal structure? We will discuss about this question later.

Another observation from the XRD data is that the strongest diffraction peak in the XRD of the monoclinic structure, (111), for all the starting powders, was the second peak in the XRD of the AGD films. This means that the AGD films have some 

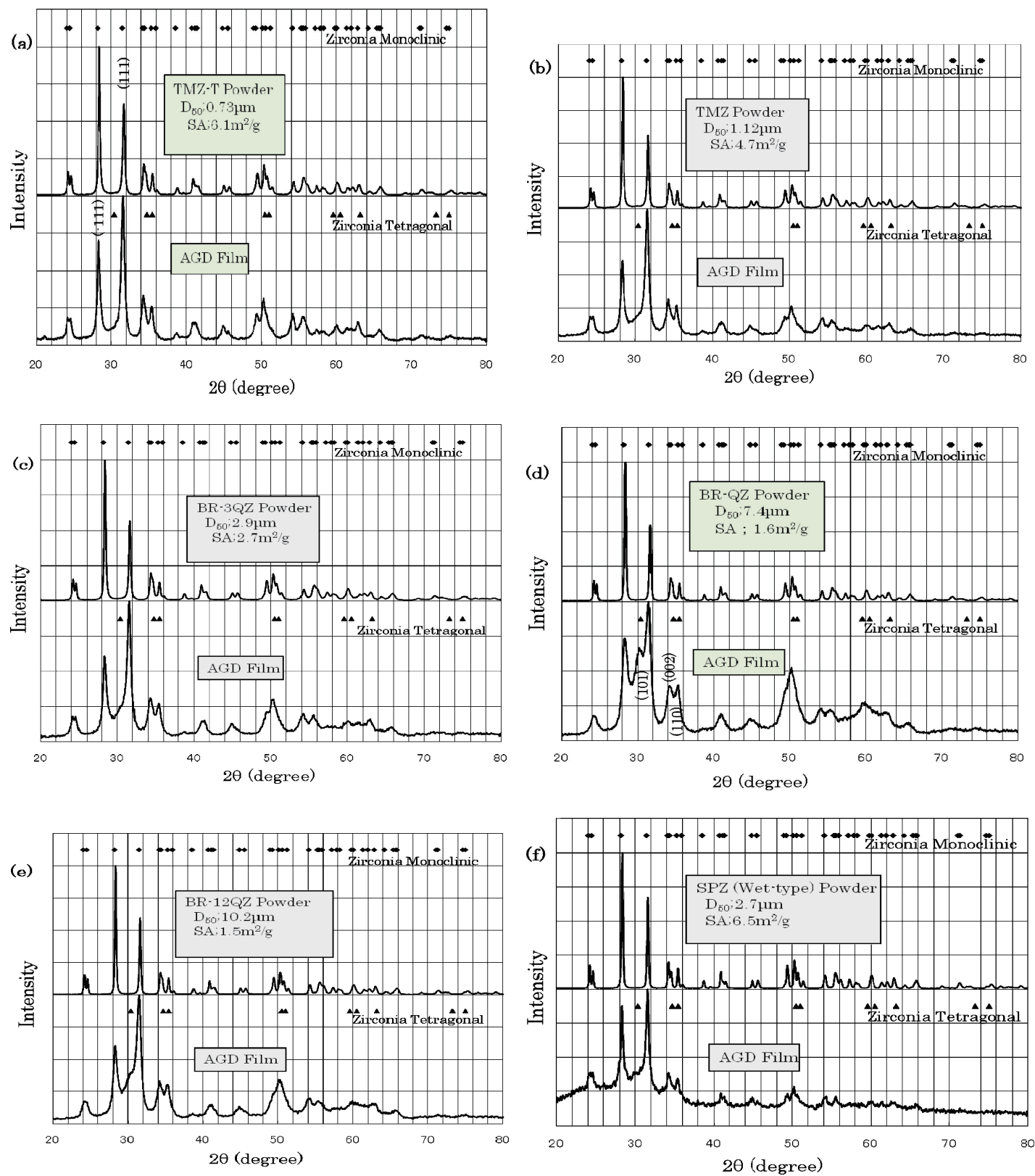

Fig. 2. (Color online) XRD figures of starting powders and AGD films. (a) TMZ-T, (b) TMZ, (c) BR-3QZ, (d) BR-QZ, (e) BR-12QZ and (f) SPZ.

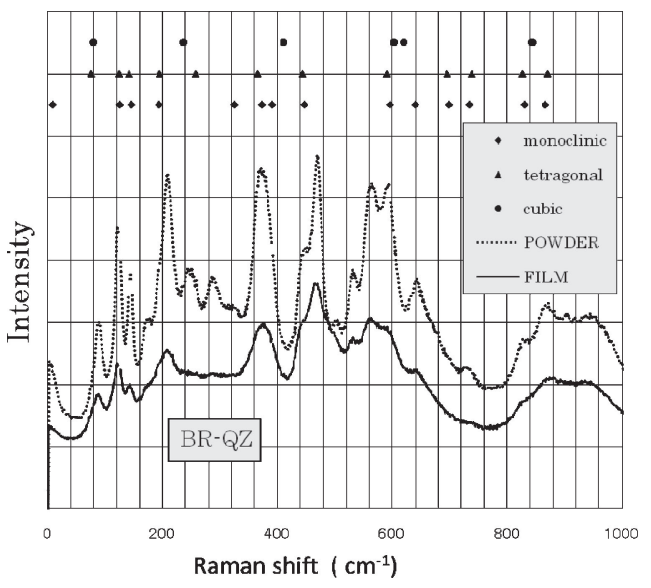

Fig. 3. Raman spectra of BR-QZ powder and the AGD film. texture. Figure 6 shows the SEM micrographs of the AGD films. We observed several ten to several hundred nanometer-size particles in each film. Because the particle size is significantly smaller than the size of the starting powders, we can conclude that the ejected powders were crushed and deposited on the substrate thus generating the texture. The texture structure of the AGD films might be formed by the alignment of the zirconia cleavage plane nearly parallel to the substrate surface. That is, the texture structure is considered to be induced by the new arrangement of the plane which is the cleavage plane. Additionally, concerning the tetragonal phase particles, we cannot identify them in the SEM micrographs. According to the previous papers, ${ }^{10)-15)}$ the appearance of the tetragonal phase was observed in the particles with less than about a $30 \mathrm{~nm}$ diameter. We could not see nearly the same size particles in every SEM micrograph of the AGD films, therefore, the tetragonal phase particles might be much smaller than the particles observed in the micrographs. 
Table 2. Ratio of the strongest peak of the tetragonal and monoclinic structure of the AGD films

\begin{tabular}{|c|c|c|c|c|c|c|c|c|}
\hline \multirow{2}{*}{ No. } & \multirow{2}{*}{$\begin{array}{c}\text { Mean particle } \\
\text { size } D_{50} \\
(\mu \mathrm{m})\end{array}$} & \multirow{2}{*}{$\begin{array}{c}\text { Specific } \\
\text { surface area } \\
\mathrm{m}^{2} / \mathrm{g}\end{array}$} & \multicolumn{2}{|c|}{ Monoclinic } & \multicolumn{2}{|c|}{ Tetragonal } & \multirow{2}{*}{$\begin{array}{c}\text { Intensity ratio } \\
\mathrm{T} / \mathrm{M}\end{array}$} & \multirow{2}{*}{ Product } \\
\hline & & & $2 \theta$ & count & $2 \theta$ & count & & \\
\hline 1 & 10.2 & 1.5 & 31.4989 & 583.63 & 30.3155 & 146.55 & 25.1 & BR-12QZ \\
\hline 2 & 7.4 & 1.6 & 31.4759 & 414.44 & 30.3620 & 315.94 & 76.2 & BR-QZ \\
\hline 3 & 3.5 & 6.1 & 31.5703 & 259.14 & 30.3161 & 28.15 & 10.9 & SPZ-N \\
\hline 4 & 2.9 & 2.7 & 31.4674 & 540.45 & 30.3506 & 46.34 & 8.6 & BR-3QZ \\
\hline 5 & 1.12 & 4.7 & 31.4761 & 592.83 & 30.2596 & 26.26 & 4.4 & TMZ \\
\hline 6 & 0.73 & 6.1 & 31.5192 & 700.62 & 30.4193 & 9.40 & 1.3 & TMZ-T \\
\hline
\end{tabular}

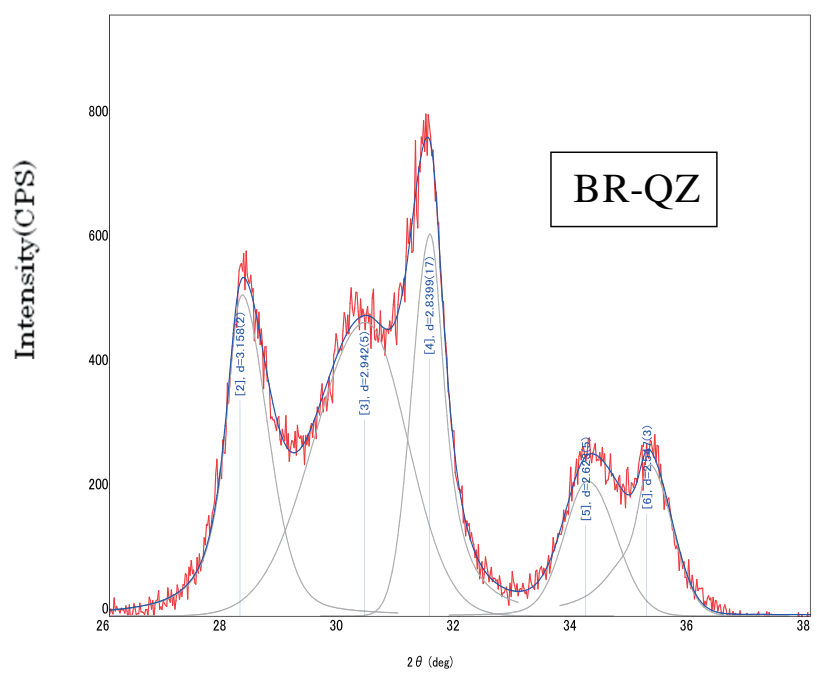

Fig. 4. (Color online) Result of a quantitative analysis for BR-QZ (Rigaku's PDXL).

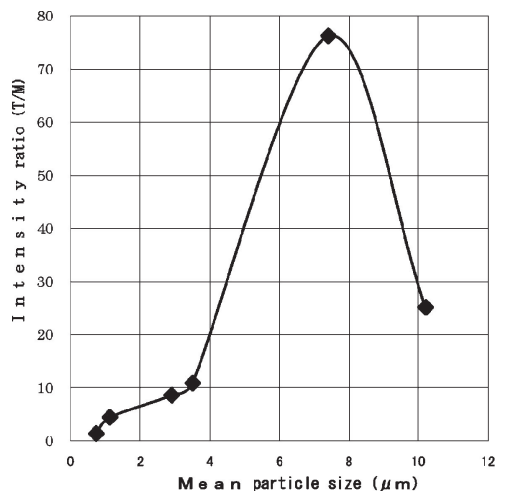

Fig. 5. Relation between the tetragonal-to-monoclinic structure ratio and the mean diameter of the starting powders.
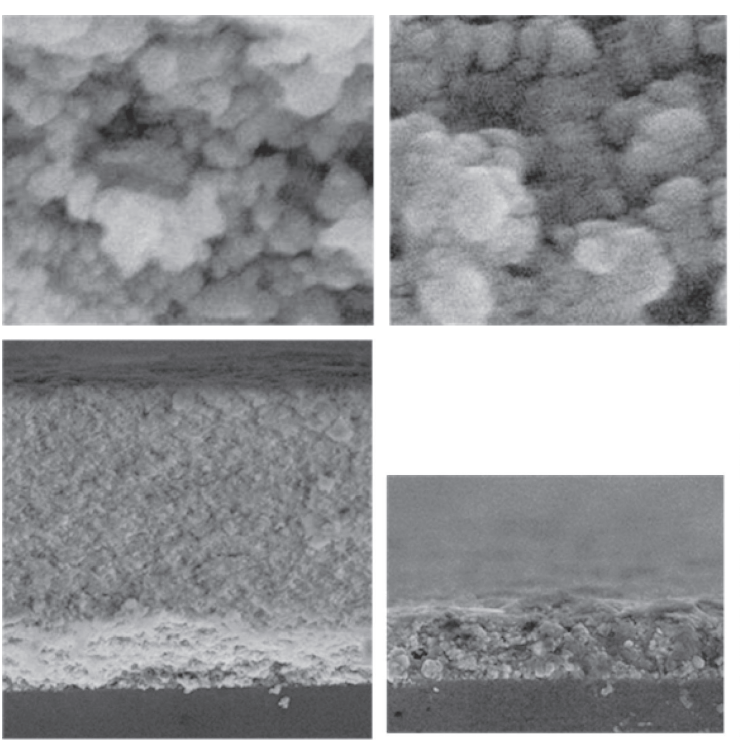

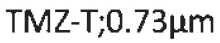

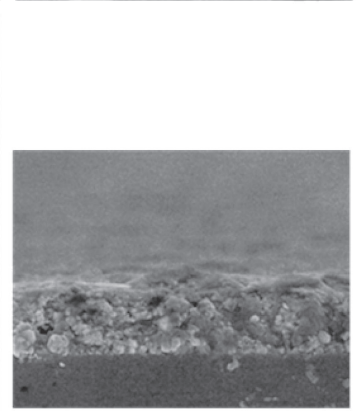

$\mathrm{TMZ} ; 1.12 \mu \mathrm{m}$
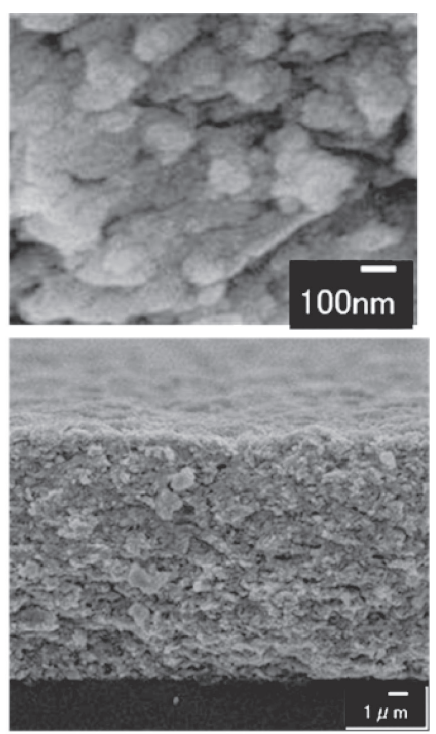

$\mathrm{BR}-\mathrm{QZ} ; 7.4 \mu \mathrm{m}$

Amount of supplied N2 gas ; $12 \mathrm{~L} / \mathrm{min}$

Number of scans; 50 pass

Scanning speed; $1 \mathrm{~mm} / \mathrm{s}$

Fig. 6. SEM micrographs of the AGD films. 


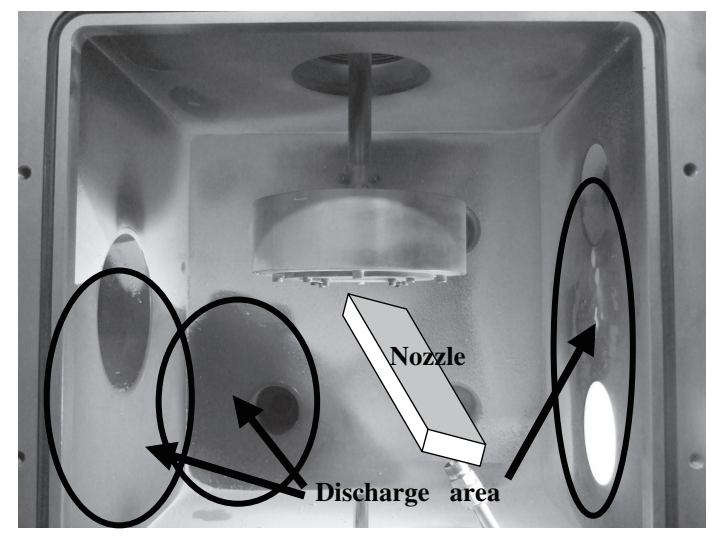

Fig. 7. (Color online) Photograph of electrical discharge phenomena on the inner chamber wall.

\subsection{Light emission form the deposition sites of the powders}

During the AGD process, we often observed a static electrical spark from the edge of the nozzle to the inner chamber wall, especially at the symmetrical sites of the chamber wall from the nozzle position, that is, the front and the back sides from the nozzle top. Figure 7 shows a signature pattern of electrical discharge phenomena on the inner chamber wall. The electrical charge is induced from the friction between the inner wall of the tubing and/or the nozzle and the source powder, and is transferred to the source powder, the tubing and the nozzle. A part of the charge transferred to the powder will be given to nitrogen molecules by collisions between the powder and nitrogen molecules. We consider that the pattern signature of the electrical discharge shown in Fig. 7 is related to the charge given to nitrogen gases. As a result, almost all the ejected powders, except for the neutral particles, will take on a positive charge. The amount of the positive charge induced on the ejected powders may depend on the powder size distribution and moisture level on the powder surface. However, the most influential factor for the charge amount might be the triboelectric series.

We found a weak light emission from the deposition site of the ejected powder on the substrate. Figure 8 shows the configuration of the nozzle and substrate and the appearance of the light emission. The light was difficult to visually see, although it was easily observed by a digital video recorder and the light sometimes had a pink color. The light emission was always seen at the deposition site of the ejected powders on the substrate, and the light emitted in a direction vertical to the substrate changed with the direction along the particle flow direction. Even if the substrate was moved in a different direction, the light emission site stayed at the same position. This light emission phenomenon is related to the powder types, the size distribution and plasma generation at the deposition site or area. The generation of plasma during the fracture of materials has been well studied. ${ }^{16)-20)}$ When some materials were fractured, fractoelectrons were emitted. The amount of the fractoelectrons depended on the materials. According to K. Yasuda et al., ${ }^{19}$ ) $\mathrm{MgO}, \mathrm{Al}_{2} \mathrm{O}_{3}, \mathrm{Si}_{3} \mathrm{~N}_{4}, \mathrm{ZrO}_{2}$ and soda lime glass formed fractoelectrons in decreasing order when they are fractured. During the AGD process, fractoelectrons and/or triboelectrons will be generated at the deposition site and the electrons will be accelerated by the positively charged powders falling from the nozzle. In order to generate the plasma, a certain amount of electrons might be needed. Since fractoelectrons come from
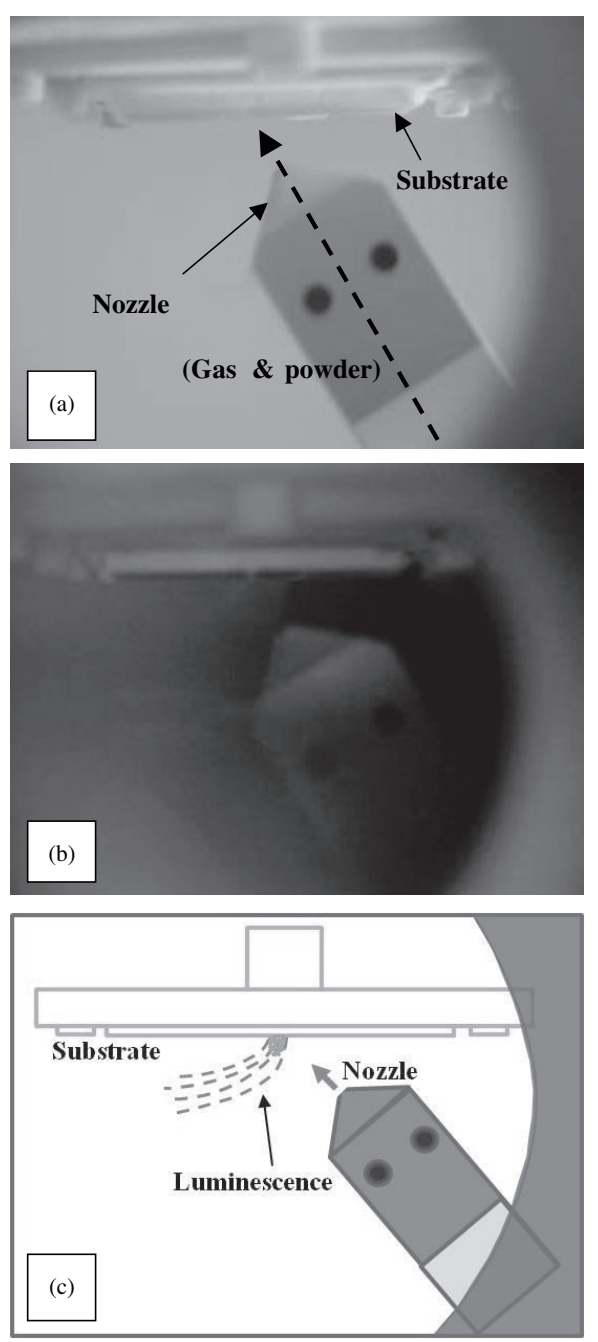

Fig. 8. (Color online) Configuration of the nozzle and substrate and the appearance of light emission. (a) Geometry of nozzle and substrate, (b) a view of light emission at a deposition site on a substrate, (c) free drawing of light emission.

fracture surfaces, the amount of the emitted electrons depends on the particle size and particle configuration as well as the particle types. In our former studies, ${ }^{8), 9)}$ we could not produce fine AGD films using powders with a high specific surface and/or more than 10-micron diameter. The results are considered to come from the decrease in the fracture surface compared to the powders thus realizing a good performance of the AGD film formation. The electrons will collide with the ambient gases which are nitrogen molecules, and form the nitrogen plasma. The light emission phenomena might be related to the nitrogen plasma. The positively charged powder and fractoelectron may play an important role in inducing the ceramic sintering during the AGD process. Even if a high voltage field does not exist, the possibility of generating a plasma still remains. K. Nakayama experimentally showed that a plasma was induced by triboelectrons emitted from a friction surface..$^{21)-24)}$ If the triboelectrons play an important role in the AGD process, we can also explain why there is a difference among the starting powders used to synthesize the AGD films. The differences among the powders in the AGD synthesis may be explained by a difference in the amount of fractoelectrons and/or triboelectron emitted from the friction or fractured surfaces of the powders. The light 
emission might have a close relation to the amount of emitted electrons. Unfortunately, we have no apparent experimental evidence for this explanation at the moment. We need more experimental data in order to continue this discussion. We will try to present more experimental data in the future in order to better understand the synthesizing mechanism of the AGD process.

\section{Conclusion}

A high temperature phase of zirconia was generated in the AGD films. The amount of the phase depended on the mean diameter of the powder particles. In our sample powders, BR-QZ with a $7.4 \mu \mathrm{m}$ diameter contained the highest amount of the hightemperature phase compared to the other powders. Light emission was also observed during synthesis of the AGD film. The luminescence will be a key factor in clarifying the AGD film formation mechanism.

Acknowledgements We would like to express our deepest gratitude to Mr. Haruki Kimura (graduate student at the University of Tsukuba), whose SEM observations were of enormous help to us. Finally, we gratefully appreciate the financial support of the New Energy and Industrial Technology Development (NEDO), which made it possible to complete our research.

\section{References}

1) S. Kasyu, E. Fuchita, T. Manabe and C. Hayashi, Jpn. J. Appl. Phys., 23, L910-L912 (1984).

2) C. Hayashi, Mater. Sci. Forum, 246, 153-180 (1997)

3) J. Akedo, J. Am. Ceram. Soc., 89, 1834-1839 (2006).

4) H. Ogawa, Mater. Trans., 47, 1945-1948 (2006).
5) Y. Imanaka, N. Hayashi, M. Takenouchi and J. Akedo, J. Eur. Ceram. Soc., 27, 2789-2795 (2007).

6) J. Akedo, J. Thermal Spray Technol., 17, 181-198 (2008).

7) T. Miyoshi, J. Ceram. Soc. Japan, 117, 899-903 (2009).

8) E. Fuchita, E. Tokizaki and Y. Sakka, J. Ceram. Soc. Japan, 118, 767-770 (2010)

9) E. Fuchita, E. Tokizaki, E. Ozawa and Y. Sakka, J. Ceram. Soc. Japan, 118, 948-951 (2010).

10) R. C. Garvie, J. Phys. Chem., 69, 1238-1243 (1965).

11) R. C. Garvie, J. Phys. Chem., 82, 218-224 (1978).

12) R. Srinvasan, C. R. Hubbard, O. B. Cavin and B. H. Davis, Chem. Mater., 5, 27-31 (1993).

13) E. Djurado, P. Bouvier and G. Lucazeau, J. Solid State Chem., 149, 399-407 (2000)

14) P. Bouvier, E. Djurado, C. Ritter, A. J. Dianoux and G. Lucazeau, Int. J. Inorg. Mater., 3, 647-654 (2001).

15) S. Shukla and S. Seal, J. Phys. Chem. B, 108, 3395-3399 (2004).

16) J. T. Dickinson, E. E. Donaldson and M. K. Park, J. Mater. Sci., 16, 2897-2908 (1981).

17) S. C. Langford, J. T. Dickinson and L. C. Jensen, J. Appl. Phys., 62, 1437-1449 (1987).

18) L. Scudiro, J. T. Dickinson and Y. Enomoto, Phys. Chem. Miner., 25, 566-573 (1998).

19) K. Yasuda, M. Shimada and Y. Matsuo, Philos. Mag. A, 82, 3251-3261 (2002)

20) T. Shiota, K. Yasuda and Y. Matsuo, Mater. Sci. Eng., B, 148, 230-233 (2008).

21) K. Nakayama, N. Suzuki and H. Hashimoto, J. Phys. D: Appl. Phys., 25, 303-308 (1992).

22) K. Nakayama and H. Hashimoto, Wear, 185, 183-188 (1995).

23) K. Nakayama and R. A. Nevshupa, Vacuum, 74, 11-17 (2004).

24) H. Tanio, S. Nakamura and T. Ohgaku, J. Mater. Sci. J., 57, 154-158 (2008). 